\title{
Treatment of high myopia/myopic astigmatism with a combination of WaveLight Contoura with LYRA protocol and wavefront-optimized treatment
}

This article was published in the following Dove Press journal:

Clinical Ophthalmology

\section{Manoj Motwani}

Motwani LASIK Institute, San Diego, CA, USA
Correspondence: Manoj Motwani

Motwani LASIK Institute, 4520

Executive Drive, Suite 230,

San Diego, CA 92121, USA

Tel +l 8585540008

Emaildrmmlj@gmail.com
Purpose: The aim of this article was to demonstrate how WaveLight Contoura and wavefront optimization (WFO) can be used together to treat high myopia/myopic astigmatism corrections. Materials and methods: A retrospective analysis was conducted on 24 consecutive myopic/ myopic astigmatism eyes that exceeded the Contoura labeling of -8.00 with $-3.00 \mathrm{D}$ of astigmatism. Residual correction after Contoura with Layer Yolked Reduction of Astigmatism protocol was treated with WFO Contoura LASIK correction. All patients had 3 months of follow-up. Accuracy to the desired refractive goal was assessed by postoperative refraction, regression, postoperative vision, and anecdotal subjective night vision quality.

Results: No eyes lost best-corrected visual acuity (BCVA), and 54\% of eyes gained BCVA. Out of 22 distance eyes, five achieved 20/15 or better, 18 achieved 20/20 vision, two achieved 20/25, and three achieved 20/30 vision. Preoperatively, only 14 eyes could achieve 20/20 vision. No night vision issues were observed in anecdotal reporting by patients except for those who needed enhancements that had not yet been performed.

Conclusion: Treatment of high myopia/astigmatism with this combination of Contoura with LYRA protocol and WFO results in excellent visual outcomes, large ablation zones on topography, and few subjective reported night vision issues.

Keywords: Contoura, topographic guided ablation, high myopia, ICL, WaveLight lasers, LYRA Protocol

\section{Introduction}

The treatment of high myopia with excimer laser has had a difficult history. Hopes for the treatment of high myopia were tempered by poor corneal optics, high amounts of night glare and halos, corneal ectasia when too much tissue was removed, and regression. ${ }^{1}$ The use of LASIK limited corneal tissue removal, and the use of photorefractive keratectomy (PRK) led to a higher amount of haze formation. ${ }^{2}$ As spherical aberration was reduced via several laser modalities, such as wavefront guided, wavefront optimized (WFO), and aspheric ablation, outcomes improved dramatically, ${ }^{3}$ but the prejudice among surgeons on performing high myopic excimer laser corrections such as $-9.00 \mathrm{D}$ and higher remained. ${ }^{4}$

Surgeons are now advocating the use of implantable contact lenses (ICLs, also called phakic intraocular lenses [IOLs] $)^{5}$ utilizing an intra-ophthalmic procedure not only for high myopic corrections but also for corrections as low as $-6.00 \mathrm{D}$ (most physicians use excimer laser ablation from 9 to $10 \mathrm{D}$ of myopia and ICL above that). ICLs themselves are fraught with risk, including cataract formation with even a small touch of the phakic lens during placement, endothelial cell loss, night vision issues, 
and a lack of long-term studies on possible negative effects of ICL to the eye. ${ }^{6-8}$ After extensive study of ICLs, we found them to be associated with too many risks of complications in comparison to laser vision correction to perform on the large majority of our high myopia patients.

Topographic-guided ablation (brand name Contoura on the WaveLight laser; WaveLight GmBH, Erlangen, Bavaria) utilizing the topography-measured refraction (Contoura with Layer Yolked Reduction of Astigmatism [LYRA] protocol) has previously demonstrated not only very high rates of better than 20/20 vision but also remarkably little induction of night glare or halos. ${ }^{9-11}$ In fact, in our clinical experience, patients have virtually no significant night glare or halos post Contoura with LYRA protocol correction.

One caveat with this approach is that the US Food and Drug Administration (FDA) approval for Contoura is only up to $-8.00 \mathrm{D}$, and up to $-3.00 \mathrm{D}$ of astigmatism, ${ }^{3}$ a relatively limited approval range. We undertook consecutive treatments with Contoura and WFO treatment of residual myopia/myopic astigmatism to determine the outcomes on procedures over $-9.00 \mathrm{D}$ of spherical equivalent correction in the FDA approval. WFO correction utilizes the average of thousands of measured eyes to add peripheral treatment to decrease spherical aberration induction with myopic ablation. Utilizing WFO after Contoura with LYRA protocol should allow a very close facsimile of a full Contoura correction, as the higherorder aberrations (HOAs) would be reduced with Contoura, the cornea made more uniform using the LYRA protocol, and the residual myopia would be treated using a spherical aberration reduction correction (WFO) that would closely model a Contoura correction following the corneal curvature.

\section{Materials and methods}

This is a retrospective analysis of the first 24 consecutive primary laser corrections of 13 patients performed by one surgeon (MM) at one center using Contoura-measured astigmatism and axis (as per the LYRA protocol) within the FDA indications of myopia up to $-8.00 \mathrm{D}$ with astigmatism no more than $-3.00 \mathrm{D}$, followed by WFO treatment of any residual myopia/myopic astigmatism, with the additional restriction that no more than a spherical equivalent of $-9.00 \mathrm{D}$ be treated on any one WaveLight EX500 treatment card. ${ }^{9-11}$ No patient was included who fitted within the treatment parameters of one Contoura treatment card, and every patient in this study required a WFO treatment on top of the Contoura treatment to fully correct their vision.

A nomogram was used to reduce spherical correction, as overcorrection can occur with high myopia treatments (Table 1). It has been the practice for many years to reduce
Table I Reduction in myopic excimer laser correction with increasing levels of myopia

\begin{tabular}{ll}
\hline $\begin{array}{l}\text { Manifest } \\
\text { sphere (D) }\end{array}$ & $\begin{array}{l}\text { Amount of laser correction } \\
\text { sphere reduction (D) }\end{array}$ \\
\hline-8.5 to -9.4 & -0.50 \\
-9.5 to -10.4 & -0.75 \\
-10.5 to -11.4 & -1.00 \\
-11.5 to -12.4 & -1.25 \\
-12.5 to -13.4 & -1.50 \\
-13.5 to -14.4 & -1.75 \\
\hline
\end{tabular}

spherical power via a nomogram when correcting over $8.50 \mathrm{D}$ of myopia. Various methodologies can be used to determine this including IBRA Outcome Planning Software (Zubisoft GmBH, Niederhasli, Switzerland), Datalink, or by the nomogram that we used (Table 1). ${ }^{12}$ This method accounts for the decrease in spherical power of correction between manifest and actual treatment.

Astigmatism was only adjusted using the Contourameasured astigmatism, and the spherical equivalent of the difference between manifest and measured was used to adjust corneal spherical power according to the LYRA protocol.

Alternatively, the IBRA surgical planning site can be used to determine this nomogram to adjust the spherical treatment. ${ }^{12}$ Adjustment for spherical equivalence due to changes in the astigmatism from manifest to Contourameasured astigmatism was performed after the decrease in the spherical power was included.

Preoperative (pre-op) corneal thickness was measured using Pentacam (OCULUS Optikgeräte GmbH, Wetzlar, Germany) to determine the thickness of the flap to be created if LASIK was performed, and if there was not enough tissue to perform a full correction with LASIK, either PRK or epi-LASIK was utilized.

LASIK was performed in 16 eyes, and corneal flaps were made either utilizing the Moria M2 microkeratome and either a $110 \mu \mathrm{m}$ (Microspecialties, Middletown, CT, USA) or $90 \mu \mathrm{m}$ head (Moria, Antony, France) or the Alcon WaveLight FS200 femtosecond laser (Wavelight GmbH, Erlangen, Germany) utilizing flap thicknesses between 90 and $110 \mu \mathrm{m}$.

Epi-LASIK was performed in six eyes using the Moria Evolution 3 Epi-LASIK system (Moria). PRK was performed in two eyes using a dilute alcohol solution and a $9 \mathrm{~mm}$ well with 30 seconds of alcohol solution exposure time. Corneal epithelium was removed using a sponge, and residual epithelium was removed using a handheld epithelium scraper. Patients undergoing surface ablation procedures had an Acuvue Oasys soft contact lens (Johnson \& Johnson Vision Care, Jacksonville, FL USA) placed to facilitate epithelial 
healing for 4-5 days after the procedure. All surface ablation procedures had adjunctive treatment with mitomycin- $\mathrm{C}$ $0.02 \%$ after laser ablation for $30-45$ seconds depending on the extent of correction performed.

All treatments were performed by utilizing the Alcon WaveLight EX500 laser (WaveLight $\mathrm{GmbH}$ ), WaveNet planning software, and the LYRA protocol. Contoura treatment optical zones were either 6.5 or $6.0 \mathrm{~mm}$ depending on the available tissue and patient pupil size. WFO treatments were either at $6.5 \mathrm{~mm}, 6.0 \mathrm{~mm}$, or $5.0 \mathrm{~mm}$ ablation size (only one patient had a $5 \mathrm{~mm}$ WFO due to tissue-sparing considerations).

We monitored all eyes for at least 3 months post LASIK via visual acuity, refraction, and Topolyzer Vario HOAs to determine whether the eyes were accurately treated. We noted any patients who complained of increased night glare and halos compared to pre-op. All surgeries were performed by one surgeon (MM) at one center in San Diego, CA, USA.

This study encompassed 24 eyes from 13 patients; of whom, five were male and eight were female. Patient ages ranged from 18 to 54 years, with an average age of 31 years. Pre-op evaluation included best-corrected visual acuity (BCVA), manifest/cycloplegic manifest refraction, anterior examination, posterior dilated examination, tonometry, pachymetry via Pentacam, auto-refraction and wavefront analysis with the Nidek OPD (Nidek Intl, Gamagori, Japan), and topographic analysis with the Topolyzer Vario.

Specific attention was paid to obtaining high-quality reproducible scans with the Topolyzer Vario. Patients had 8-12 scans performed per eye, and at least four accurate similar scans with appropriate iris registration and complete data (as indicated by the Topolyzer Vario) were necessary to proceed in surgical planning. If the scans were too variable, or not enough scans with high-quality information were taken, the scans were repeated until enough scans were obtained to create an accurate, reproducible, consistent average scan. Care was taken not to induce astigmatism when holding the eyelids open for scans, and blinks were allowed to prevent the corneal surface from drying out.

Patients were not included if they had prior refractive surgery, had anterior segment abnormalities, or findings that could affect the outcome such as keratoconus or corneal ectasia, recurring eye disease such as iritis, or hepatitis, uncontrolled diabetes or hypertension, were pregnant, or had severe dry eye.

All patients signed consents that allowed their data and records to be used anonymously for research study and publication. This study conforms with the exemption under HHS Policy for Protection of Human Research Subjects 45 CFR
46.101 (b) for retrospective studies and therefore did not require institutional review board approval.

\section{Results}

Results were evaluated for the amount of correction performed, regression or change that occurred by 3 months, final vision, improvement or loss of lines of BCVA, and anecdotal complaints of increased night glare/halos/night vision issues. This latter category is strictly emphasized as anecdotal, and no standardized subjective patient survey was utilized. For the purposes of this analysis, all manifest refractions were dilated refractions using tropicamide $1 \%$ to reduce the impact of accommodation.

The average of pre-op manifest refraction treated was $-10.41 \mathrm{D}$ of myopia and $-1.83 \mathrm{D}$ of cylinder. The average treatment amount was $-8.68 \mathrm{D}$ of myopia and $-1.76 \mathrm{D}$ of cylinder. The spherical equivalent of the manifest refraction was $-10.80 \mathrm{D}$, and the average spherical equivalent of laser treatment was $-9.55 \mathrm{D}$. The range of manifest myopia treated was from -7.50 to $-15.5 \mathrm{D}$. The range of manifest astigmatism treated was from -0.25 to $-3.75 \mathrm{D}$. The range of Contourameasured astigmatism treated was from -0.25 to $-4.80 \mathrm{D}$.

The average difference between manifest astigmatic axis and Contoura-measured astigmatic axis was $10.55^{\circ}$, with the range of difference from $0^{\circ}$ to $68^{\circ}$. The average amount of difference between the magnitude of astigmatism from manifest refraction astigmatism to Contoura-measured astigmatism was $0.6895 \mathrm{D}$, with a range of $0-3.3 \mathrm{D}$. This number differs from the average amount of abovementioned correction as some eyes had no astigmatism present on manifest refraction, but astigmatism was present on Contoura-measured refraction. Every patient had astigmatism treated, even though two eyes did not have astigmatism on manifest refraction but did have astigmatism on Contoura-measured refraction.

No eyes lost BCVA, and 13 eyes (54.17\%) gained lines of BCVA. A total of 11 eyes gained one line of BCVA, one eye gained two lines, and one eye gained three lines.

Out of 22 distance eyes (two were monovision eyes), five achieved $20 / 15$ vision, 18 achieved 20/20 vision, two achieved 20/25 vision (one needed a small enhancement not performed at the time of this writing to achieve 20/20; one had a BCVA of 20/25 preoperatively), and three achieved $20 / 30$ vision (all three of these were amblyopic eyes that had improvements of BCVA of $+1,+1,+3$ lines, respectively). Four of the eyes that achieved 20/20 vision could not achieve 20/20 preoperatively; they had gained one line of BCVA. Every single eye that could achieve 20/20 vision did except for the one that needed enhancement to achieve 20/20. 
Amblyopia in this group was significant. Ten out of 24 (41.67\%) eyes could not achieve $20 / 20$ vision. Nine out of $10(90 \%)$ eyes gained at least one line of BCVA at 3 months postoperatively.

Postoperatively, 18 eyes achieved a BCVA of 20/20 (as opposed to 14 preoperatively). Of these 18 eyes, 17 (94.4\%) were $20 / 20$ or better uncorrected, and five (27.84\%) were $20 / 15$ or better. One eye was $20 / 25$ in this group and required a small correction to achieve 20/20 which had not been performed at the time of this writing.

Seven of $24(20.83 \%)$ eyes needed a second correction performed 3 months after the procedure to achieve the pre-op goal for correction. The manifest refractions were as follows:

- $-0.75,-1.50 \times 120$

- $-0.50,-1.75 \times 105$

- $+0.75,-1.25 \times 105$ (secondary correction performed as Contoura $+0.50,-0.34 \times 152$ )

- Plano, $0-0.75 \times 12$

- $-0.75,-1.50 \times 105$

- $+1.00,-1.25 \times 85-$ uncorrected OD

- $-0.25,-1.25 \times 105$ - uncorrected OS

There were similarities in the second corrections; all had astigmatism, which is what we see in most Contourabased regression; and all had very similar axis of correction. Secondary treatments were performed with WFO correction or repeat Contoura topographic guided ablation in case the patient had a peri-pupillary coma causing the astigmatism.

\section{Sample cases}

Sample case I: LASIK performed on 19-year-old

female with I year of follow-up data

- Pre-op dark pupil size: $4.5 \mathrm{~mm}$ OU Pre-op manifest:

- OD: $-10.75,-0.25 \times 170$

- OS: $-11.50,-0.75 \times 105$ Measured treatment:

- OD: Contoura: $-8.00,-0.43 \times 5$ at $6.5 \mathrm{~mm}$ optical zone (OZ); WFO: -1.75 at $6.5 \mathrm{~mm} \mathrm{OZ}$

- OS: Contoura: $-8.00,-0.25 \times 114$ at $6.5 \mathrm{~mm} \mathrm{OZ}$; WFO: -2.25 at $6.5 \mathrm{~mm} \mathrm{OZ}$

- Postoperative (Post-op) vision at 1 week, 3 months, 6 months, and 1 year: 20/15 OU with plano refraction.

\section{Sample case 2: epi-LASIK performed on 22-year-old} female with 4 months of follow-up data

- Pre-op dark pupil size: $6 \mathrm{~mm}$ OU Pre-op manifest:

- OD: $-13.50,-3.25 \times 175, \mathrm{BCVA}=20 / 40$
- OS: $15.50,-3.75 \times 15, \mathrm{BCVA}=20 / 50$

Measured treatment:

- OD: Contoura: $-7.75,-2.30 \times 1$ at $6.0 \mathrm{~mm} \mathrm{OZ}$; WFO: -4.75 at $5.0 \mathrm{~mm} \mathrm{OZ}$

- OS: Contoura: $-7.50,-2.87 \times 7$ at $6.0 \mathrm{~mm} \mathrm{OZ}$; WFO: -6.00 at $5.0 \mathrm{~mm} \mathrm{OZ}$

- Post-op vision at 4 months: $20 / 30+$ OU with plano refraction.

Sample case 3: epi-LASIK OU performed on 53-yearold female with 10 months of follow-up data

- Pre-op dark pupil size: $5 \mathrm{~mm}$ OU Manifest refraction:

- OD: $-13.50,-2.50 \times 180 ; \mathrm{BCVA}=20 / 40-$

- OS: $-13.25,-2.50 \times 10 ; \mathrm{BCVA}=20 / 25$

Measured correction:

- OD: Contoura: $-8.0,-1.37 \times 173$ at $6.0 \mathrm{~mm} \mathrm{OZ}$; WFO: -2.75 at $6.0 \mathrm{~mm} \mathrm{OZ}$

- OS: Contoura: $-8.0,-1.78 \times 14$ at $6.0 \mathrm{~mm} \mathrm{OZ}$; WFO: -3.75 at $6.0 \mathrm{~mm} \mathrm{OZ}$

- One enhancement performed OS at 6 months: WFO: plano, $-0.75 \times 12$ at $5.0 \mathrm{~mm} \mathrm{OZ}$

- Post-op vision at 10 months: 20/40 OD (mono -1.50, BCVA = 20/25); 20/20 OS.

Sample case 4: PRK performed on 25-year-old male with 3 months of follow-up data

- Pre-op dark pupil size: $6 \mathrm{~mm} \mathrm{OU}$ Pre-op manifest (undilated):

- OD: $-10.25,-2.75 \times 1 \mathrm{BCVA}=20 / 30$

- OS: $-10.50,-3.50 \times 178 \mathrm{BCVA}=20 / 30$ Pre-op manifest (cyclopleged):

- OD: $-9.50,-1.50 \times 180 \mathrm{BCVA}=20 / 25$

- OS: $-10.25,-1.50 \times 5 \mathrm{BCVA}=20 / 25$ Measured Contoura correction:

- OD: Contoura: $-7.00,-3.00 \times 179$ at $6.0 \mathrm{~mm} \mathrm{OZ}$; WFO: plano, $-1.75 \times 179$ at $6 \mathrm{~mm} \mathrm{OZ}$

- OS: Contoura: $-7.50,-2.91 \times 4$ at $6 \mathrm{~mm} \mathrm{OZ}$; WFO: -1.00 at $6 \mathrm{~mm} \mathrm{OZ}$

- Post-op vision at 3 months: 20/20 OU with plano refraction OU

- Post-op vision at 4 months: 20/30+ OU with plano refraction.

\section{Discussion}

Patients with high myopia (disclaimer: the author himself was a -10 D myope) have had a limited number of choices for treatment. Small ablation zones and significant 
induced spherical aberration caused significant issues with night vision and dashed the hopes of many who wished to have their disabling levels of myopia/myopic astigmatism corrected. ICLs have been proposed as the procedure for higher myopic corrections, but not only do they have significant complications, ${ }^{6-8}$ they do nothing to remove the HOAs of the cornea allowing for better vision and decreased night glare issues.

Topographic-guided ablation utilizing the topographymeasured refraction, ie, Contoura-measured astigmatism and use of the LYRA protocol holds the promise of being the ideal correction for high myopes - virtual elimination of spherical aberration, wide smooth ablation zones, reduction in HOAs, and resultant good day and night vision..$^{9-11}$ Indeed, in this particular cohort of patients, only one patient reported night glare/halo issues in both eyes. This patient was lost to follow up, as she never scheduled her secondary correction even though the patient is now more than one year postoperative. We are reasonably confident that removing her astigmatism in both eyes would have resolved the night issues. One other patient reported night vision difficulty in one of her eyes. She had a small amount of astigmatism in one eye, which was corrected, but this patient was also lost to any longterm follow-up. When she came in post-op she was found to have a partial retinal detachment which resulted in retinal surgery, and further data were not available as of the writing of this manuscript.

In fact, although we did not do formal subjective patient surveys, anecdotally this cohort had no other patients after the initial healing period of the first month postoperatively who complained that their night vision worsened or even complained of night glare/halo issues. This was the second most striking feature postoperatively of these patients; the most striking was the large, smooth ablation zone that structurally matched with the lack of patient visual complaints. This removal of HOAs and creation of a more uniform cornea would also seem to explain the number of eyes that gained lines of vision (54\%) and the lack of any eyes with a loss of BCVA.

Treatment of high myopia with this protocol was remarkably effective, accurate, and stable. The optics created by such a uniform cornea led to the rather remarkable anecdotal result of only two patients in this group complaining of increase in night vision halos or glare, or even in any night vision difficulties at all, and both were patients who needed an enhancement "regressed" with astigmatism. A large number of patients had a gain of lines of BCVA, and none had loss of any lines of BCVA. More eyes achieved 20/20 vision than could pre-op, and the lack of lines lost is critical in showing that good optics can be achieved with even a large correction. More eyes achieved a BCVA of 20/20 postoperatively than preoperatively, demonstrating that even with amblyopia, improvements in lines of visual acuity can be attained with the removal of HOAs and creation of a more uniform cornea. About $54 \%$ of eyes gained lines of BCVA, with none losing lines, demonstrating how important a uniform cornea is to correcting these high myopia patients.

Post-op topographic analysis showed a large flat, fairly uniform optical zone (Figures 1-4). We believe this more uniform optical zone creates the optics that prevent the scatter of light and is likely responsible for good night vision.

In sample case 1, a 19-year-old female achieved 20/15 vision within a week after her procedure and showed the large, smooth, uniform ablation zone as shown in Figure 1 on virtually every topographic map taken from 1 week onward. She also had 20/15 vision almost immediately and had no problems with night vision at a week even though we corrected $11 \mathrm{D}$ of myopia.

In the sample case 2, we took a more creative approach with her very high myopia and astigmatism, which limited the amount of tissue available for ablation. The original Contoura correction was performed at $6 \mathrm{~mm}$ and included all the astigmatism treatment, and the residual myopia $(-2.75 \mathrm{OD},-3.75 \mathrm{OS})$ was treated with WFO with a smaller $5 \mathrm{~mm}$ zone creating a "stepped" appearance to the topography. This allowed us to complete the entire correction utilizing epi-LASIK leaving behind a safe amount of corneal bed tissue. We postulated that this two-zone correction should not dramatically negatively affect the night vision, and the patient preferred full correction with some risk of night vision issues. This seems to have been successful as she had 20/30+ vision in both eyes (a gain of +1 and +2 lines) and has no complaints about her night vision.

Sample case 3 had to be performed as epi-LASIK as she did not have sufficient corneal thickness to perform her correction via LASIK. Nevertheless, even though she had a slower healing curve, she also did very well with very smooth, wide ablation zones on both topographies. She had a small astigmatic enhancement to her left (distance) eye, and her bilateral amblyopia actually improved by one line on the distance eye and two lines on her monovision eye. She did need a small enhancement on her distance eye.

Sample case 4 is an interesting case of a young man who had high myopia and astigmatism, with the undilated manifest refraction being similar to his autorefraction and 

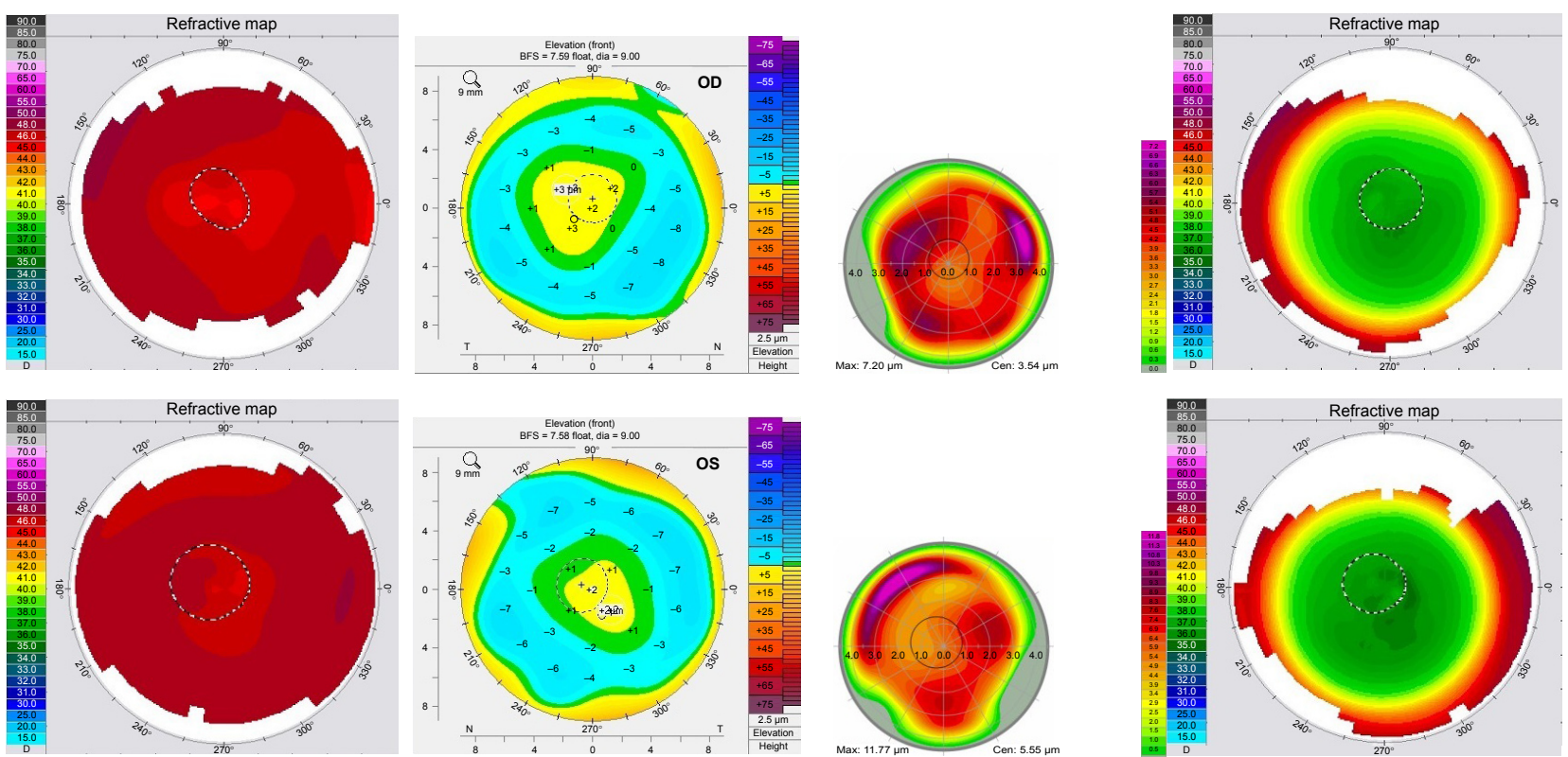

Figure I OD and OS Topolyzer topographic scans pre-op, Pentacam anterior elevation pre-op, HOAs pre-op, post-op I year Topolyzer scans for Sample case I. Abbreviations: HOAs, higher-order aberrations; pre-op, preoperative; post-op, postoperative.

glasses. He was having severe allergic reactions to his contacts, was simply unable to wear them any longer, and was wearing his thick glasses. His cycloplegic refraction showed a far lower amount of astigmatism, but refracted him preoperatively to a BCVA of a line better demonstrating the effects that HOAs can even have on refraction depending on pupil size. His Contoura-measured refraction was quite a bit higher, but closer again to his undilated manifest.
Not only did this patient achieve plano correction in both eyes, he ended up gaining a line of vision on each side and achieved 20/20 vision in each eye by 2 months postoperatively. Interestingly enough, he even measured at 20/15 at one point at about 6 weeks postoperatively in one eye. This patient was also interesting in another way; he started out as an epi-LASIK, but his corneas were too small to create a proper epithelial flap. He ended up needing PRK in both
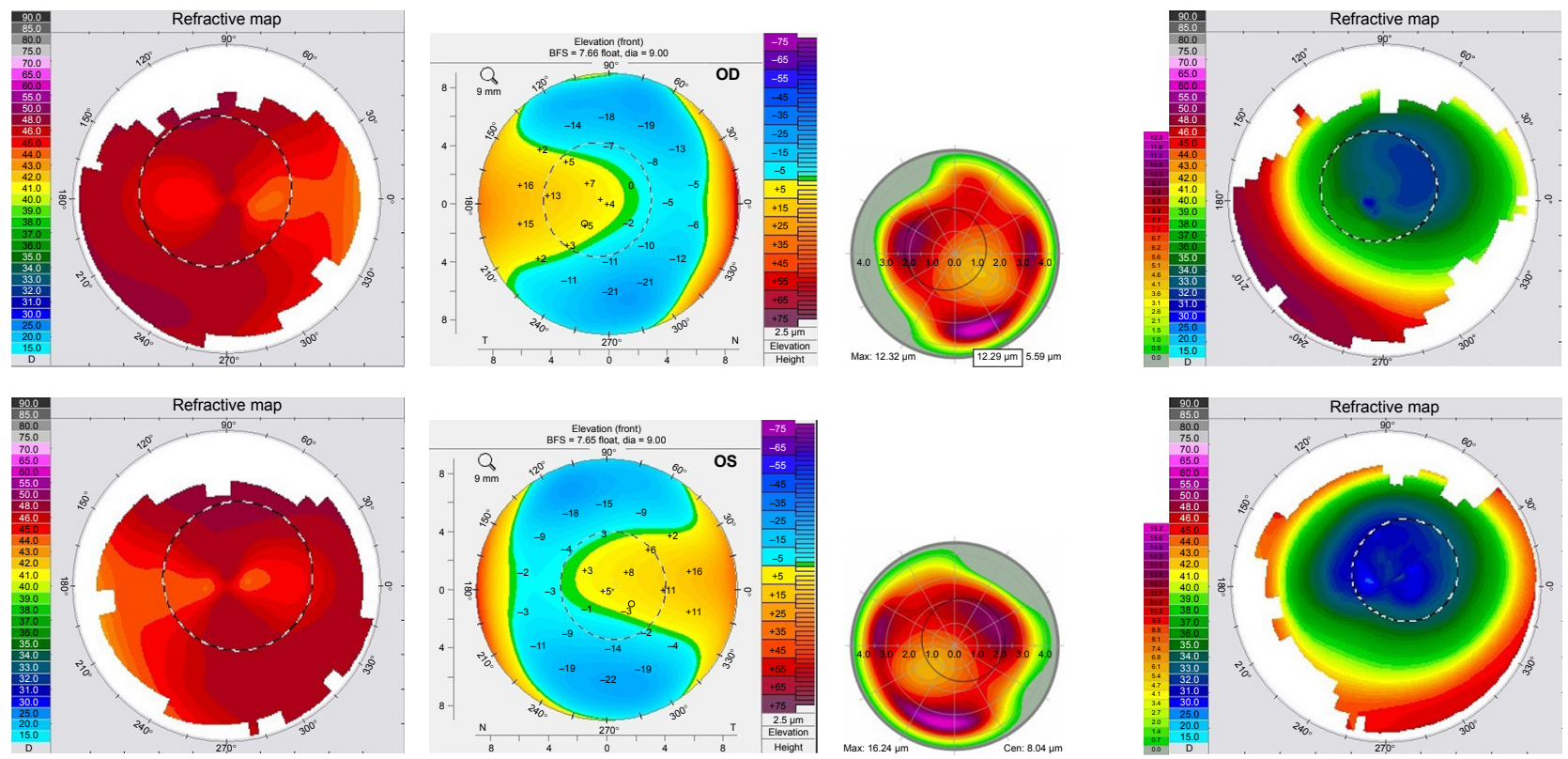

Figure 2 OD and OS Topolyzer topography scans pre-op, Pentacam anterior elevation pre-op, HOAs pre-op, post-op 4 month Topolyzer scans for Sample case 2. Abbreviations: HOAs, higher-order aberrations; pre-op, preoperative; post-op, postoperative. 

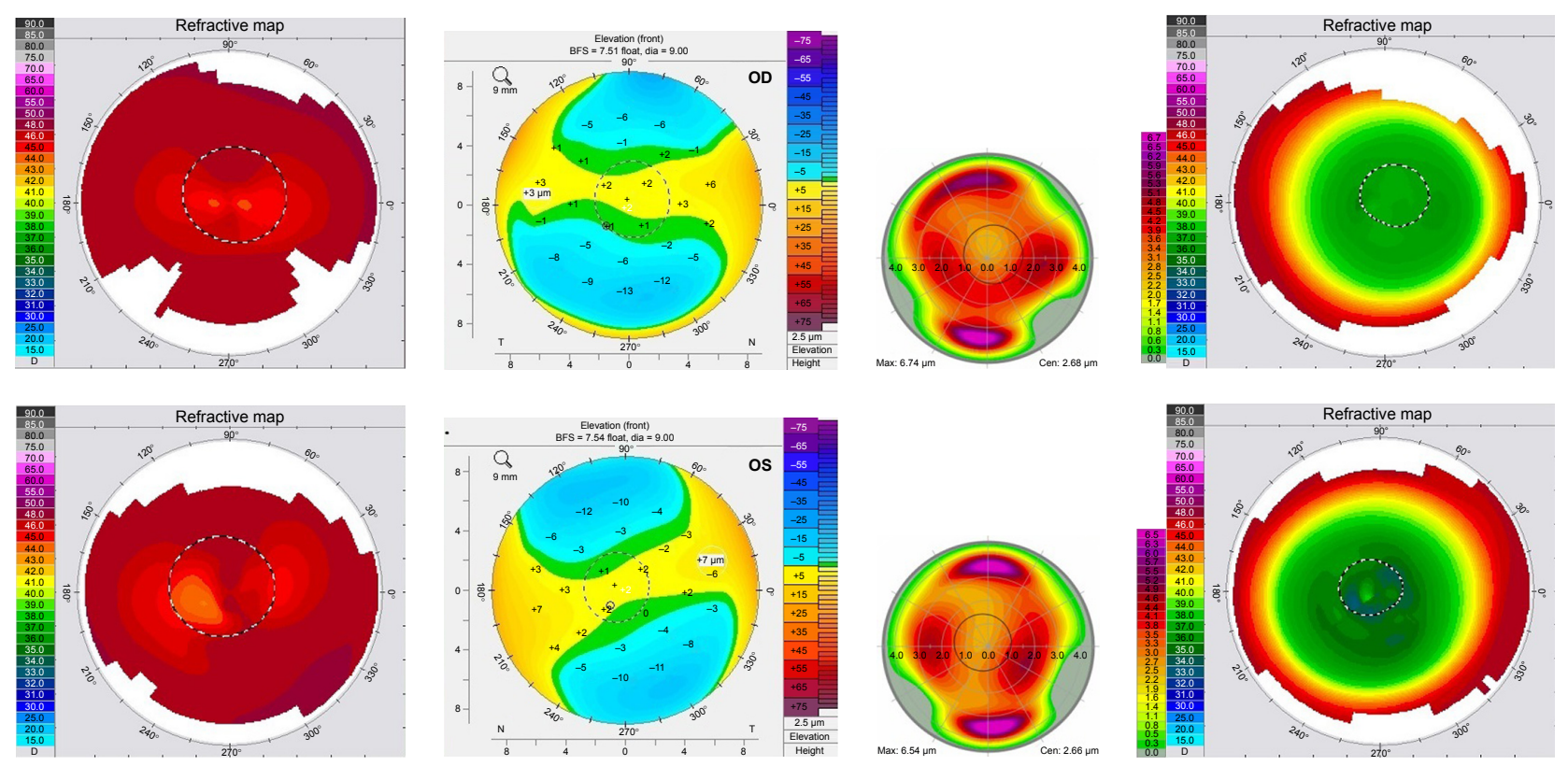

Figure 3 OD and OS Topolyzer topography scans pre-op, Pentacam anterior elevation pre-op, HOAs pre-op, post-op 10 months Topolyzer scans for Sample case 3. Abbreviations: HOAs, higher-order aberrations; pre-op, preoperative; post-op, postoperative.

eyes. Interestingly, even with his high corrections no haze occurred. He did undergo 45 seconds of mitomycin-C $0.02 \%$ treatment.

The types of second corrections necessary in this cohort were virtually all astigmatic in nature, which coincides with our theory that residual HOAs were hidden by epithelial compensation preoperatively. We are undertaking a study to use epithelial thickness mapping via optical coherence tomography (OCT) to determine how HOAs partially masked by epithelial compensation can lead to "regression" and secondary corrections.

In comparison to ICL placement, no risk of cataract, endophthalmitis, or endothelial cell loss exists, and since a more uniform cornea is made with the reduction in HOAs, it stands to reason that the subjective visual outcomes could be better. The only way to really answer this question would
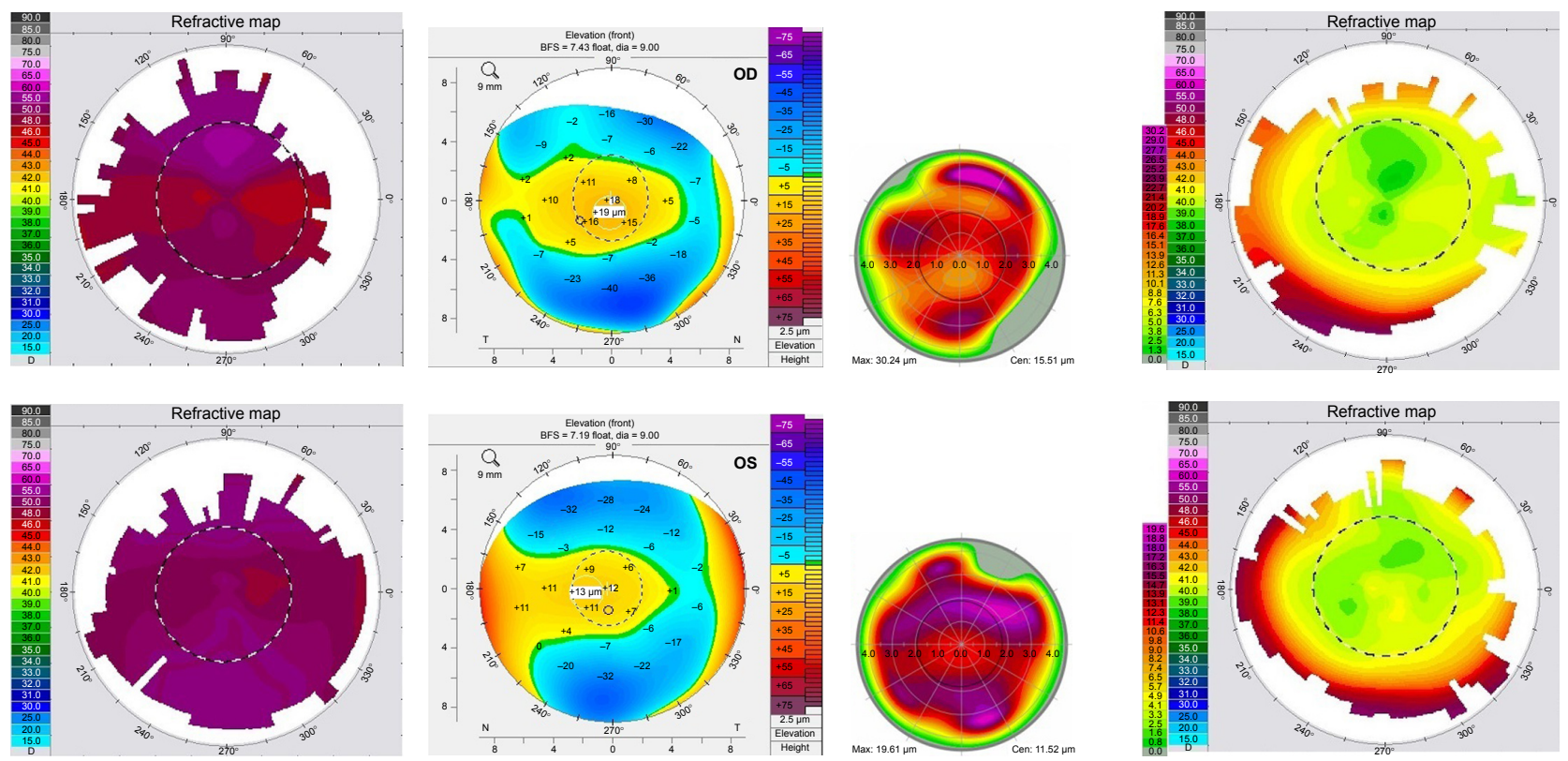

Figure 4 OD and OS Topolyzer topography scans pre-op, Pentacam anterior elevation pre-op, HOAs pre-op, post-op 3 months Topolyzer scans for Sample case 4. Abbreviations: HOAs, higher-order aberrations; pre-op, preoperative; post-op, postoperative. 
be a contralateral ICL/laser ablation study with objective and subjective visual outcomes.

The main risk with this procedure is corneal ectasia if the amount of tissue removal is not carefully calculated leaving too thin a bed. ${ }^{2}$ Although most refractive surgeons leave at least $250 \mu \mathrm{m}$ in the bed, with these procedures we calculated the best way was to leave a bed of approximately $290 \mu \mathrm{m}$, to not only leave an extra measure of safety but also leave some tissues if retreatment was necessary. The use of epi-Lasik for high correction cases as a tissue-sparing procedure has been very successful in this group, with minimal to no haze formation. None of the patients had to have a procedure to remove corneal haze nor ended up with haze as a visionlimiting issue. The marked lack of significant haze in the surface ablation patients has left us with the thought that perhaps aberration reduction and a more uniform cornea somehow helps prevent haze formation, but this is merely an observation in a limited cadre of eyes.

One patient did have a retinal detachment shortly after an enhancement, but this was several months after the original procedure. The retinal surgeon I discussed the case with did not see how raising a flap and performing laser ablation could lead to enough manipulation to damage the cornea and thought this was an unfortunate coincidence. It serves to remind us that these high myopia patients are at higher risk for retinal complications, ${ }^{13}$ a risk that may be even higher with patients having intra-ophthalmic procedures such as ICL or lens exchange.

The main issue with using this treatment paradigm changing technology to help these patients has been the limited FDA approval obtained by Alcon Surgical (Fort Worth, TX, USA) on the WaveLight Contoura approval. It is unfortunate that this critical advancement was not initially available to these patients who can derive significant benefit from it, but via the use of consecutive WFO treatment of the residual correction we can closely mimic a Contoura correction of the full amount of myopia/myopic astigmatism with the main drawback being the use of a second treatment card. Although we are unaware of further FDA approval studies initiated to expand the approval ranges for Contoura, the authors hope that Alcon Surgical will consider doing so in the future.

A note on FDA labeling, the question can fairly be raised if this is an off-label procedure. WFO for myopia is FDA approved, as is Contoura. Is the combination together considered off-label? Germaine to this issue is that the Contoura retreatment FDA labeling is that of a precaution, but not expressly off-label. Therefore, WFO could be considered the primary procedure, and Contoura retreatment the consecutive procedure and this could theoretically be on-label. This could also just be considered the use of two FDA approved procedures used consecutively. There are differing opinions on this issue, and we fall on the side that this is on-label.

We understand the enormous challenges patients with high myopia face. Without correction, the patient is virtually completely functionally disabled, and in emergency situations if glasses or contacts are not available, the consequences for the patient can be severe. The ability to help these patients is crucial.

This procedure may very well be the high correction procedure of choice. If care is taken with carefully calculating tissue removal depths, the results are predictable, the visual outcomes are very good, and the night vision issues present with excimer laser ablation of high myopia in the past seem largely absent. There has been a significant shift over the past decade to lens-based solutions in refractive surgery. With topographic-guided ablation, the ability to make supranormal corneas has changed the equation, and further work on corneal solutions for large refractive corrections should be performed, as this may be the ideal way to correct high myopia/myopic astigmatism.

\section{Acknowledgment}

The author would like to acknowledge Sissimonis Lemonis of WaveLight $\mathrm{GmBH}$ for the inspiration to conduct this analysis.

\section{Disclosure}

The author has received grants from Alcon, Inc, Dermasciences, Inc, and Acufocus, Inc for unrelated studies. The author reports no other conflicts of interest in this work.

\section{References}

1. El Bahrawy M, Alio JL. Excimer laser 6(th) generation: state of the art and refractive surgical outcomes. Eye Vis (Lond). 2015;2:6.

2. Azar DT, Chang JH, Han KY. Wound healing after keratorefractive surgery: review of biological and optical considerations. Cornea. 2012; 31(suppl 1):S9-S19.

3. Moshirfar M, Shah TJ, Skanchy DF, Linn SH, Kang P, Durrie DS. Comparison and analysis of FDA reported visual outcomes of the three latest platforms for LASIK: wavefront guided Visx iDesign, topography guided WaveLight Allegro Contoura, and topography guided Nidek EC-5000 CATz. Clin Ophthalmol. 2017;11:135-147.

4. Motwani M, Pei R. Treatment of moderate-to-high hyperopia with the WaveLight Allegretto 400 and EX500 excimer laser systems. Clin Ophthalmol. 2017;11:999-1007.

5. Solomon R, Donnenfeld ED. Refractive intraocular lenses: multifocal and phakic IOLs. Int Ophthalmol Clin. 2006;46(3):123-143.

6. Chen LJ, Chang YJ, Kuo JC, Rajagopal R, Azar DT. Metaanalysis of cataract development after phakic intraocular lens surgery. J Cataract Refract Surg. 2008;34(7):1181-1200.

7. Sanders DR. Actual and theoretical risks for visual loss following use of the implantable contact lens for moderate to high myopia. J Cataract Refract Surg. 2003;29(7):1323-1332. 
8. Ieong A, Hau SC, Rubin GS, Allan BD. Quality of life in high myopia before and after implantable Collamer lens implantation. Ophthalmology. 2010;117:2295-2300.

9. Motwani M. The use of WaveLight(R) Contoura to create a uniform cornea: the LYRA Protocol. Part 1: the effect of higher-order corneal aberrations on refractive astigmatism. Clin Ophthalmol. 2017;11: 897-905.

10. Motwani M. The use of WaveLight(R) Contoura to create a uniform cornea: the LYRA Protocol. Part 2: the consequences of treating astigmatism on an incorrect axis via excimer laser. Clin Ophthalmol. 2017;11:907-913.
11. Motwani M. The use of WaveLight(R) Contoura to create a uniform cornea: the LYRA Protocol. Part 3: the results of 50 treated eyes. Clin Ophthalmol. 2017;11:915-921.

12. Seven I, Vahdati A, De Stefano VS, Krueger RR, Dupps WJ Jr. Comparison of patient-specific computational modeling predictions and clinical outcomes of LASIK for myopia. Invest Ophthalmol Vis Sci. 2016;57(14):6287-6297.

13. Chen E, Regillo CD. Retinal complication of cataract and refractive surgery. Retinal Phys. 2007. Available from: https:/www.retinalphysician.com/issues/2007/april-2007/retinal-complications-of-cataract-andrefractive-s. Accessed April 1, 2017.

\section{Publish your work in this journal}

Clinical Ophthalmology is an international, peer-reviewed journal covering all subspecialties within ophthalmology. Key topics include: Optometry; Visual science; Pharmacology and drug therapy in eye diseases; Basic Sciences; Primary and Secondary eye care; Patient Safety and Quality of Care Improvements. This journal is indexed on

Submit your manuscript here: http://www.dovepress.com/clinical-ophthalmology-journal

\section{Dovepress}

PubMed Central and CAS, and is the official journal of The Society of Clinical Ophthalmology (SCO). The manuscript management system is completely online and includes a very quick and fair peer-review system, which is all easy to use. Visit http://www.dovepress.com/ testimonials.php to read real quotes from published authors. 